\title{
Multiresolution Texture Analysis of Surface Reflection Images
}

\author{
Leena Lepistö ${ }^{1}$, Iivari Kunttu ${ }^{1}$, Jorma Autio ${ }^{2}$, and Ari Visa ${ }^{1}$ \\ ${ }^{1}$ Tampere University of Technology, Institute of Signal Processing \\ P.O. Box 553, FIN-33101 Tampere, Finland \\ \{Leena.Lepisto, Iivari.Kunttu, Ari.Visa\}@tut.fi \\ http: //www.tut.fi \\ ${ }^{2}$ Saanio \& Riekkola Consulting Engineers \\ Laulukuja 4, FIN-00420 Helsinki, Finland \\ Jorma.Autio@sroy.fi \\ http: //www.sroy.fi
}

\begin{abstract}
Surface reflection can be used as one quality assurance procedure to inspect the defects, cracking, and other irregularities occurring on a polished surface. In this paper, we present a novel approach to the detection of defects based on analysis of surface reflection images. In this approach, the surface image is analyzed using texture analysis based on Gabor-filtering. Gabor-filters can be used in the inspection of the surface in multiple resolutions, which makes it possible to inspect the defects of different sizes. The orientation of the defects and surface cracking is measured by applying the Gabor-filters in several orientations. A set of experiments were carried out by using surface reflection images of polished rock plates and the orientation of the surface cracking was determined. In addition, the homogeneity of the rock surface was measured based on the Gabor features. The results of the experiments show that Gabor features are effective in the measurement of the surface properties.
\end{abstract}

\section{Introduction}

During recent years, the number of industrial imaging systems has increased remarkably. The purpose of these digital imaging solutions is often the control of quality or production. In these solutions, analysis of the image data is made by using some image processing method. One typical application for the image analysis system is to detect and analyze the defects occurring in the production. Image analysis is used to detect possible malfunctioning as soon as possible to minimize the economic losses. Another application is classification of the products in different categories.

Surface reflection is a phenomenon that can be utilized in the detection of defects and microfracturing on different surfaces. In addition, based on the surface reflection, other surface properties can also be analyzed. These properties can be for example uniformity and smoothness of the surface.

The reflection image obtained from the surface can be analyzed using methods and tools developed in the field of texture analysis. The majority of the texture analysis 
methods are based on the statistical textural features, some texture model, or application of the signal processing tools. An example of the statistical tools is co-occurrence matrix [3], whereas Multiresolution autoregressive model (MRSAR) [10] represents the model-based features. Recently, the methods based on signal processing have been widely used in the analysis of textures. Commonly used filtering-based signal processing methods use wavelets [4] or Gabor features [9]. The benefit of the wavelet-based methods is that they measure the textural properties in multiple resolutions. Manjunath and Ma [9] have made a comparison between multiple oriented Gabor filters, other wavelet-features, and MRSAR-model. In this comparison, the best results were achieved using Gabor-filtering.

In rock industry, the digital imaging tools are used in the quality and production control of rock. Rock is a commonly used as ornamental stones in the building industry, where rock plates are used e.g. to cover the floors and walls of buildings. When the plates are used in the external walls, they are required to tolerate different weather conditions. The cracking and other defects occurring in the surface of the rock plates have significant effect on their strength and ability to bear frost and moisture. Therefore, it is beneficial for a rock manufacturer to be able to classify and assure the quality of the plates. Important properties of the rock surface are strength and directionality of the surface cracking. The homogeneity of the surface reflection is also important, because the smoothness of the polished surface can be determined based on its homogeneity.

In the rock image analysis, it has been made several studies about the rock texture images. Autio et al. [1] have researched rock texture characterization and classification using co-occurrence matrix and texture directionality. In [6] we presented a classification system for non-homogenous rock images using textural and spectral features. The texture directionality was used in rock image classification in [7]. All these studies concern the images of rock texture, whereas the number of the researches about the surface reflection is very limited. However, in [5] surface reflection model for rock is presented. This study was focused mainly on image acquisition and the methods used for the reflection image analysis were not discussed.

In this paper, we use multiscale texture analysis methods for the inspection of the surface reflection images. We use the textural properties to distinguish between homogenous and non-homogenous surfaces. For testing purposes, we used a set of industrial rock plates, whose surface reflection was used in testing of analysis methods.

\section{Analysis of Surface Reflection Images}

When the light approaches a surface at an angle $\Theta_{1}$ to the normal of the surface, a part of it reflects from the surface at the same angle $\Theta_{2}$ at the opposite side of the normal (figure 1a). If the angle $\Theta_{1}$ exceeds a certain critical angle $\Theta_{c}$, all the approaching light reflects from the surface. This phenomenon is called total reflection, in which the surface acts like a mirror reflecting the light at angle $\Theta_{2}$. This reflection can be utilized in the surface inspection, because the light reflects from the smooth surface in 

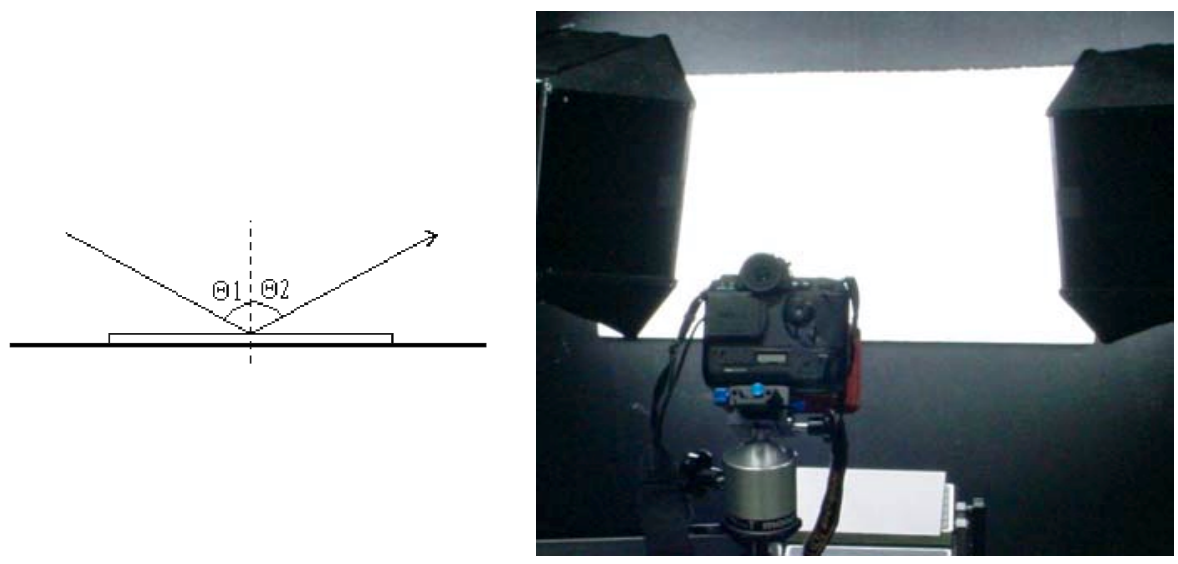

Fig. 1. a) The surface reflection model, b) The imaging arrangement of the rock plates

a different way than from the cracking and other defects. Using a sophisticated digital camera system, this reflection pattern can be acquired into digital form, in which it can be processed and analyzed.

\subsection{Multiresolution Texture Analysis}

Directionality of the cracking and the other surface properties can be described using texture analysis. By means of multiscale texture analysis, the surface structures can be analyzed in the several resolutions. This is essential, because the size of the surface cracks and defects may vary strongly.

The analysis methods presented in this paper use the texture representation based on the Gabor filters. The experimental results of [9] show that Gabor filtering gives the best texture classification compared to the other, commonly used wavelet-based texture analysis tools. Gabor filters can also be considered as tunable edge and line (bar) detectors [8]. This property can be utilized in the description of the surface cracking. The Gabor-features used in this study are based on the work of Manjunath and Ma [9]. They have used a bank of Gabor filters to characterize the texture properties. This filter bank can be used in multiple scales and orientations. Gabor function is based on the gaussian wavelet function [2]. A two dimensional Gabor function $g(x, y)$ and its Fourier transform $G(u, v)$ can be written as [9]:

$$
\begin{gathered}
g(x, y)=\left(\frac{1}{2 \pi \sigma_{x} \sigma_{y}}\right) \exp \left[-\frac{1}{2}\left(\frac{x^{2}}{\sigma_{x}^{2}}+\frac{y^{2}}{\sigma_{y}^{2}}\right)+2 \pi j W x\right] \\
G(u, v)=\exp \left\{-\frac{1}{2}\left[\left(\frac{(u-W)^{2}}{\sigma_{u}^{2}}+\frac{v^{2}}{\sigma_{v}^{2}}\right)\right]\right\}
\end{gathered}
$$


where $\sigma_{u}=1 / 2 \pi \sigma_{x}$ and $\sigma_{v}=1 / 2 \pi \sigma_{y}$. Let $g(x, y)$ be a mother Gabor wavelet, then filter at multiple rotations and scales can be obtained by appropriate dilations and rotations of $g(x, y)$ through the generating function:

$$
\begin{aligned}
& g_{m n}(x, y)=a^{-m} G\left(x^{\prime}, y^{\prime}\right), \quad a>1, m, n=\text { integer } \\
& x^{\prime}=a^{-m}(x \cos \theta+y \sin \theta), \text { and } y^{\prime}=a^{-m}(-x \sin \theta+y \cos \theta)
\end{aligned}
$$

where $\Theta=n \pi / K, K$ is the total number of orientations, and $a^{-m}$ is an energy factor that ensures that energy is independent of $m$ [9]. When we have an image $\mathbf{I}(x, y)$, its Gabor wavelet transform is defined as [9]:

$$
W_{m n}(x, y)=\int \mathbf{I}\left(x_{1}, y_{1}\right) g_{m n} *\left(x-x_{1}, y-y_{1}\right) d x_{1} d y_{1}
$$

where * indicates the complex conjugate.

\subsection{Textural Description of the Surface Reflection Images}

The multiscale texture representation presented in section 2.1 can be used to characterize the surface reflection image. An effective textural descriptor for a texture region is the mean of the transform coefficient magnitudes for the scale $m$ and the orientation $n$ [9]:

$$
\mu_{m n}=\iint\left|W_{m n}(x, y)\right| d x d y
$$

Using $\mu_{m n}$, the distribution of the orientations occurring in the image can be formed for each scale. Mean value $\mu_{m n}$ can be defined for a set of scales $[M]=s_{1}, s_{2}, \ldots, s_{k}$, and for the orientations $[N]=\Theta_{1}, \Theta_{2}, \ldots, \Theta_{l}$. Then the dominating orientation $\Theta_{D}$ at the scale $m$ is the orientation, in which $\mu_{m n}$ has its maximum value. When $\mu_{m n}$ is defined for all $k$ scales and $l$ orientations of the sets $[M]$ and $[N]$, a feature vector for a texture region can be defined as:

$$
\mathbf{F}=\left[\mu_{00}, \mu_{01}, \mu_{02}, \ldots, \mu_{k l}\right]
$$

Using this feature vector, texture properties of a texture region can be compared with the properties of the other regions.

In addition to the orientation of the surface cracking, also homogeneity of the surface is essential in the rock surface analysis. The mean of the transform coefficients $\mu_{m n}$ can be used also in the measurement of the texture homogeneity. In this case, the reflection image is divided into $B$ subimages (or blocks). The textural properties of $i$ :th block are measured by calculating a feature vector $\mathbf{F}_{i}$ for it. Then the average feature vector $\mathbf{F}_{\text {ave }}$ of the all $B$ blocks in the image is defined. 


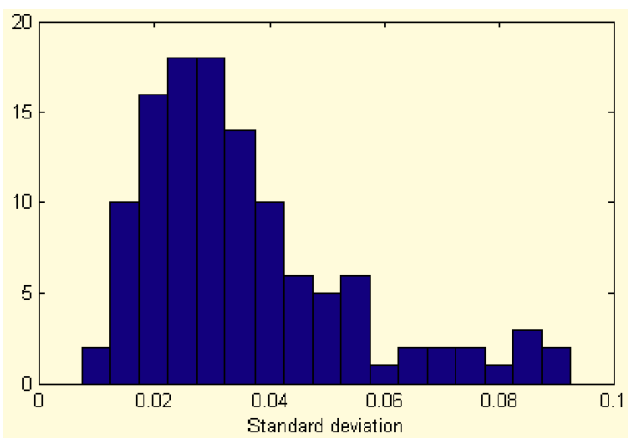

Fig. 2. The distribution of the standard deviation (std) values of the test set images
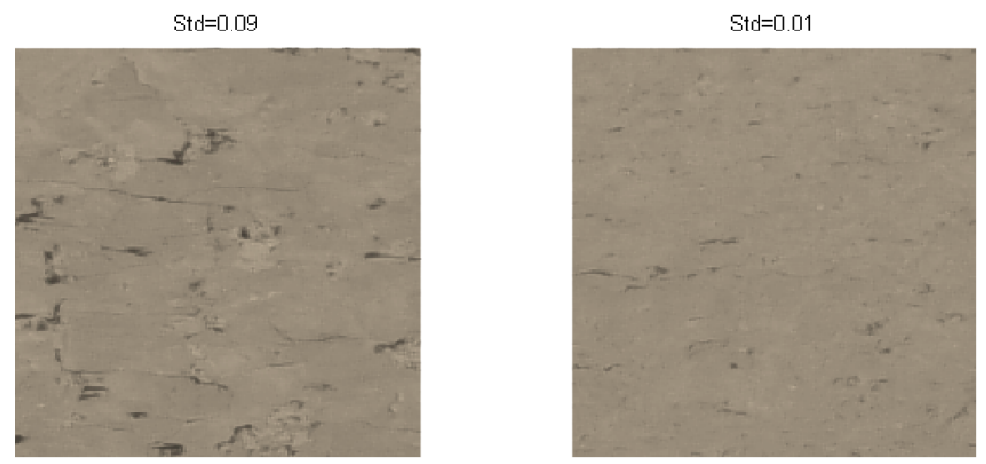

Fig. 3. Example images of non-homogenous and homogenous reflection images

The homogeneity of the image can be measured by means of the deviation between $\mathbf{F}_{i}:$ s and $\mathbf{F}_{\text {ave }}$. Hence, in homogeneity measurement we use standard deviation $($ std $)$ :

$$
s t d=\frac{1}{B} \sqrt{\sum_{i=1}^{B} D_{i}^{2}}, \text { in which } D_{i}=\sum_{i=1}^{k l} \mathbf{F}_{a v e}(i i)-\mathbf{F}_{i}(i i)
$$

Standard deviation is a measure that describes the texture homogeneity.

\section{Experiments}

For testing purposes we had 118 polished industrial rock plates. The surface of each plate was photographed using a digital camera combined with polarization filter. The imaging arrangement is presented in figure $1 \mathrm{~b}$. In this arrangement, a plane of fluorescence tubes illuminated the plate via a white vertical surface. Using this lightning method, the plate surface was evenly illuminated. 
We applied to the obtained surface reflection images the texture analysis methods presented in section 2.2. An area of 500x500 pixels was selected from the middle of the plate to represent the surface of the plate. For this region, Gabor wavelet transform of equation 4 was defined using a set of four scales and six orientations. Using these scales and orientations, the transform coefficients $\mu_{m n}$ were calculated. Based on these coefficients, the dominating orientation of each plate was defined. Based on the manual inspection of the reflection images, the results of the orientation measurements were valid.

Another goal was to measure the homogeneity of the surface. For this purpose, the images were divided into 25 blocks so that the size of each block was 100x100 pixels. The feature vector $\mathbf{F}_{i}$ of equation 6 was defined for each block of each image. Then the standard deviation (std) between the block feature vectors (equation 7) was calculated for each image. The manual inspection of the images showed that there is a clear relation between the $s t d$-value and the homogeneity of the surface image. Hence, the low value of std means that the surface is homogenous whereas large std-value indicates non-homogeneity of the surface. The distribution of the std-values of the images is presented in figure 2. Based on this distribution, the thresholds for $s t d$-values of homogenous and non-homogenous rock plates can be defined. In figure 3, examples of non-homogenous and homogenous surface reflection images are presented.

\section{Discussion}

In this paper we presented a novel method for the analysis of the surface reflection images. This method is based on the multiscale texture analysis, which makes it possible to analyze the orientations and other textural properties in multiple resolutions. The benefit of this approach is that the defects and microcracks of different sizes can be found.

The application field of this method lies in the rock and stone industry. The surface inspection by image analysis is beneficial in the quality control of the rock plates. It is fast and accurate compared to traditional visual methods. The directionality of surface cracking of the rock plate is an important feature to inspect. On the other hand, the homogeneity of the surface reflection indicates the average smoothness and void content of the plate. Therefore, we applied the surface analysis method to two purposes: directionality and homogeneity measurement. The experimental results show that both of these properties can be inspected from the images using the methods presented in this paper. The method made it possible to classify the test material accurately based on both of these two properties.

The surface reflection method proved to be feasible in the analysis of the rock plate surfaces. The method for the image acquisition is straightforward and the texture analysis tools were able to analyze the desired features in the images. The obtained results show that this method has great potential in rock and stone industry. However, these methods can also be applied to other surface inspection tasks. 


\section{Acknowledgment}

We would like to thank Saanio \& Riekkola Consulting Engineers and the Technology Development Centre of Finland (TEKES's grant 40397/01) for financial support.

\section{References}

1. Autio, J., Luukkanen, S., Rantanen, L., Visa, A.: The Classification and Characterization of Rock Using Texture Analysis by Co-occurrence Matrices and the Hough Transform. International Symposium on Imaging Applications in Geology, Belgium (1999) 5-8

2. Chui, C.K.: An Introduction to Wavelets. Wavelet Analysis and Its Applications, Vol. 1. Academic press, London (1992)

3. Haralick, R.M., Shanmugam, K., Dinstein, I.: Textural Features for Image Classification. IEEE Transactions on Systems, Man, and Cybernetics, Vol. SMC-3, 6 (1973) 610-621

4. Laine, A. Fan, J.: Texture Classification by Wavelet Packet Signature. IEEE Transactions on Pattern Analysis and Machine Intelligence, Vol. 15, 11 (1993) 1186-1191

5. Lebrun, V.: Development of Specific Image Acquisition Techniques for Field Imaging Applications to Outcrops and Marbles-. International Symposium on Imaging Applications in Geology, Belgium (1999) 165-168

6. Lepistö, L., Kunttu, I., Autio, J., Visa, A.: Rock Image Classification Using NonHomogenous Textures and Spectral Imaging. WSCG Short papers proceedings, WSCG'2003, Plzen, Czech Republic (2003) 82-86

7. Lepistö, L., Kunttu, I., Autio, J., Visa, A.: Retrieval of Non-Homogenous Textures Based on Directionality. Proceedings of the $4^{\text {th }}$ European Workshop on Image Analysis for Multimedia Interactive Services, London, UK (2003) 107-110

8. Manjunath, B.S., Chellappa, R.: A Unified Approach to Boundary Detection. IEEE Transactions on Neural Networks, Vol. 4, 1 (1993) 96-108

9. Manjunath, B.S., Ma, W.Y.: Texture Features for Browsing and Retrieval of image Data. IEEE Transactions on Pattern Analysis and Machine Intelligence, Vol. 18, 8 (1996) 837 842

10. Mao, J., Jain, A.K.: Texture Classification and Segmentation using Multiresolution Simultaneous Autoregressive Models. Pattern Recognition, Vol. 25, 2 (1992) 173-188 